\title{
Numerical Calculation of Electric Fields in Housing Spaces due to Electromagnetic Radiation from Antennas for Mobile Communication
}

\author{
H.-P. Geromiller, A. Farschtschi
}

The influence of electromagnetic radiation from mobile antennas on humans is under discussion in various group of scientists. This paper deals with the impact of electromagnetic radiation in housing spaces. The space is assumed to be bordered by 5 walls of ferroconcrete and a door-window combination on the $6^{\text {th }}$ side, the latter to be electromagnetic transparent. The transparent side of the housing is exposed to an electromagnetic wave. As the source of radiation is considered to be far away from the housing, the radiation is regarded as a plane wave. Due to the high signal frequency and the ferroconcrete walls, 5 sides of the housing space are considered to be perfect conductors. The electric field inside the housing is calculated numerically by the method of finite differences for different angles of incidence of the radiated electromagnetic wave. The maximum value of the calculated electric field is outlined in a diagram.

Keywords: numerical calculation, finite difference, electric fields, mobile communication.

\section{Introduction}

The aim of this paper is to calculate the electric fields caused by the impact of electromagnetic waves inside a housing space. The calculation is performed numerically with the finite-difference time-domain method (FDTD). The method of finite differences is based on discretisation of infinite small time and space steps by finite small time and space steps. The code applied for this investigation approximates the timeand space dependences of magnetic $(H)$ and electric $(E)-$ fields by central differencies.

Eq. 1 a-c shows discretisation along dimensions $(x, y, z)$ of the Cartesian grid, Eq. 2 shows discretisation of time.

$$
\begin{aligned}
& \frac{\partial}{\partial x} F^{n}(x, y, z)=\frac{F^{n}\left(x+\frac{1}{2}, y, z\right)-F^{n}\left(x-\frac{1}{2}, y, z\right)}{\Delta x} \\
& \frac{\partial}{\partial y} F^{n}(x, y, z)=\frac{F^{n}\left(x, y+\frac{1}{2}, z\right)-F^{n}\left(x, y-\frac{1}{2}, z\right)}{\Delta y} \\
& \frac{\partial}{\partial z} F^{n}(x, y, z)=\frac{F^{n}\left(x, y, z+\frac{1}{2}\right)-F^{n}\left(x, y, z-\frac{1}{2}\right)}{\Delta z} \\
& \frac{\partial}{\partial t} F^{n}(x, y, z)=\frac{F^{n+1 / 2}(x, y, z)-F^{n-1 / 2}(x, y, z)}{\Delta t} \\
& F: \quad \quad \quad E, H, \text { respectively, } \\
& n: \quad \text { time step, } \\
& x, y, z: \quad \text { location in Cartesian dimensions. }
\end{aligned}
$$

$$
\begin{aligned}
& \alpha: \begin{array}{l}
\text { angle of } \\
\text { incidence }
\end{array} \\
& \mathrm{k}: \begin{array}{c}
\text { direction of } \\
\text { propagation }
\end{array}
\end{aligned}
$$

a) cutting of the vector of the incident wave $\mathrm{E}_{\text {inc }}$ into components $\mathrm{E}_{\text {inc }}(\mathrm{z})$ and $E_{\text {inc }(y)}$ on sourceplane

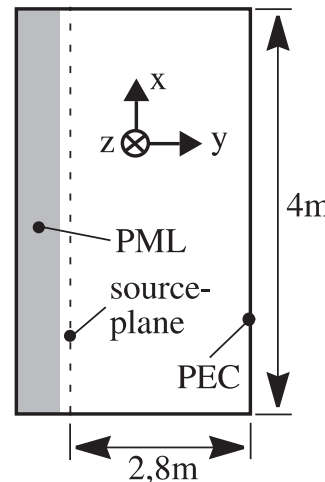

b) side-view on housing-space

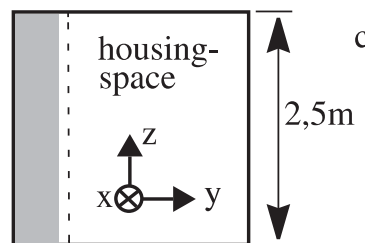

c) top-view on housing-space

Fig. 1: Simulation of wave propagation and housing space dimensions 
As only electromagnetic waves are considered, Maxwell's equations are approximated by omitting the current density.

FDTD requires the computational domain to be limited by boundary conditions. Two boundary conditions are used here, perfectly electric conductors (PEC) and perfectly matched layers (PML). PECs are implemented by forcing the tangential component of the electric field along the boundaries to be zero $\left(E_{\text {tangential }}=0\right)$. In the PML technique, an artificial layer of absorbing material is placed around the outer boundary of the computational domain. The goal is to ensure an electromagnetic wave incident into the PML-region at an arbitrary angel to be absorbed without reflection. The PML region is realized by implementing a new degree of freedom into the formulas of the FDTD code, which is done by splitting the field components [1].

Fig. 1 shows the dimensions of the house spacing under consideration from a top and side view.

The house spacing under consideration is restricted to a volume of $V=4 \mathrm{~m} \times 2.8 \mathrm{~m} \times 2.5 \mathrm{~m}$. Five boundaries are considered to be walls of ferroconcrete and therefore good reflectors for high-frequency signals as used for mobile communication. Hence these walls are simulated with PECs. The $6^{\text {th }}$ boundary is considered to be a window/door combination and therefore electromagnetically transparent. In order to simulate the incidence of electromagnetic waves from radiating antennas far away from the housing, the window/door boundary is simulated as a source plane. For calculation, the direction of propagation needs to be taken into account, so the electric field vector of the incident wave is simulated on the source plane by decomposition into components. Simulation is performed by setting the components of the electric field strength $E_{i n c(z)}$ and $E_{i n c(y)}$ on the source plane according to the electric field strength $E_{i n c}$ of the incident electromagnetic wave on the window/door combination. Time dependence is taken into account by setting the values of the components of the electric field strength on the source plane sinusoidally. The setting of the magnetic component was omitted, as magnetic and electric fields are related by the impedance of free space. Impedance matched simulation of the free space along the window/door combination (source plane) is assured by implementing PMLs. The PML structure numerically absorbs the energy of the electromagnetic wave traveling from the interior of the house spacing towards the environment.

\section{Results}

As shown in Fig. 1a, numerical calculation was performed for an electromagnetic wave with magnetic and electric field vectors $H_{i n c}$ and $E_{i n c}$ and direction of propagation $k$ related to the dimensions of the defined Cartesian system. Investigation of electric field strength inside the house-spacing was based on different angles of incidence within the range of $5^{\circ} \leq \alpha \leq 85^{\circ}$ in steps of $\alpha_{\text {step }}=5^{\circ}$. For each angle of incidence, the calculation was performed until a steady state of the electric field inside the housing space could be observed.

Data analysis was restricted to the last time inverval in a steady state. The last time interval was divided into 10 time points with equal time-spacing.

Fixing the angle of incidence of the propagating electromagnetic wave, the maximum absolute value of the electric field strength $E_{\max }$ was detected within the housing space and within the chosen time points for steady state. In addition $E_{\text {max }}$ was referred to the amplitude of the incident electric field strength $E_{i n c}$.

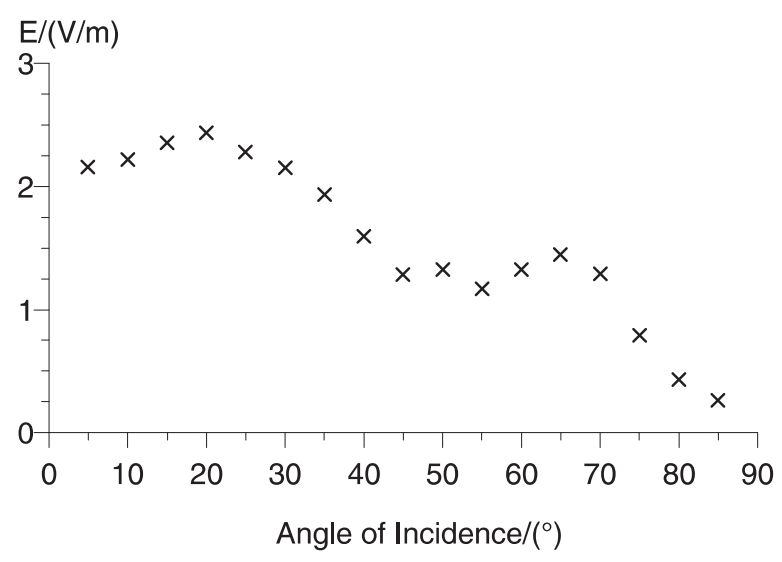

Fig. 2: Dependency of maximum electric field strength inside a housing space referred to the incident electric field strength $\left(E_{\max } / E_{\text {inc }}\right)$ over angle of incidence $(\alpha)$

Fig. 2 shows $E_{\max } / E_{\text {inc }}$ over $\alpha$. As may be seen, the maximum electric field strength depends strongly on the angle of the incident electromagnetic wave and the maximum value of the electric field strength inside the housing exceeds the value of the electric field strength of the incident wave. The maximum may be observed for $\alpha=20^{\circ}$ with a ratio

$$
\frac{E_{\max }}{E_{\text {inc }}} \approx 2.5
$$

This may be explained by reflections and superposition on the perfectly conducting walls of the house spacing, particulary in corners and edges where supercomposition with reflected electric fields from several walls may occur. Taking into account the density of electromagnetic energy being quadratically dependent on the electric field strength, it may be argued that the density of energy may in the worst case be about 5 times higher than the energy density of the incident electromagnetic wave on the source plane.

\section{Conclusion}

From Fig. 2 it may be conlcuded, that the effects of electromagnetic radiation from antennas for mobile communication should not only be judged by their electric field strength in free space or boundaries between free space and housings. As electromagnetic waves with high frequencies may have negative effects on humans, attention should be paid to legal limits for electromagnetic radiation from radio transmitters for mobile communication in the vicinity of housings. Legal limits referring to free space propagation of electromagnetic waves should be regarded with care, since unfavourable conditions inside housings may subject humans to electric field strengths exceeding the allowed limits. In this context it should be taken into account that the effects of electromagnetic radiation on humans are quadratically dependent on electric field strength, as these effects are mainly 
related to the energetic density of electromagnetic waves, and therefore the negative impacts on humans increases disproportionately with electric field strength.

\section{References}

[1] Berenger J. P.: "A perfectly matched layer for the Absorption of Electromagnetic Waves". Journal of computational Physics, Vol. 114 (1994), p.185-200.

[2] Sadiku M.: Numerical Techniques in Electromagnetics. CRC-Press, 2001, Second Edition, ISBN 0-8493-13953, p. 121-186.

[3] Simonyi K.: Theoretische Elektrotechnik. Deutscher Verlag der Wissenschaften, 10. Auflage, ISBN 3-335-00375-60.

Dr.-Ing. H.-P. Geromiller

phone: +493715313354

fax.: +493715313417

email: hans-peter.geromiller@e-technik.tu-chemnitz.de

Prof. Dr.-Ing. habil. A. Farschtschi

Technical University of Chemnitz

Chair of Fundamentals of Electromagnetics

09111 Chemnitz, Germany 